\title{
Effects of Ultrasound Section-Thickness on Brachytherapy Needle Tip Localization Error
}

\author{
Mohammad Peikari, Thomas Kuiran Chen, Andras Lasso, \\ Tamas Heffter, and Gabor Fichtinger
}

School of Computing, Queen's University, Kingston, Ontario, K7L 3N6, Canada \{mpeikari, chent, lasso, heffter, gabor\}@cs.queensu.ca

\begin{abstract}
Purpose: Ultrasound section-thickness is the out-of-plane beamwidth causing major roles in creating image artifacts normally appearing around the anechoic areas. These artifacts can introduce errors in localizing the needle tips during any ultrasound-guided procedure. To study how section-thickness and imaging parameters can affect observing and localizing needle tips, we have conducted a typical calibration setup experiment. Method: Multiple needles were inserted orthogonal to the axial image plane, at various distances from the transducer. The experiment was conducted on a brachytherapy stepper for a curvilinear transrectal-ultrasound probe. Result: Experiments demonstrated that the imaging parameters have direct impacts on observing needle tips at different axial locations. They suggest specific settings to minimize the imaging artifacts. Conclusion: The ultrasound section-thickness and side lobes could result in misjudgment of needle insertion depth in an ultrasound-guided procedure. A beam profile could assist in considering the likelihood of position errors, when the effects of side lobes are minimized.
\end{abstract}

\section{Introduction}

Ultrasound (US) imaging is ubiquitous in intra-operative surgical guidance. It has been discussed in the literature [1 15$]$ that the US images may contain certain artifacts caused by the section-thickness (elevation beamwidth) of the beam, orthogonal to both the axial and lateral beam axes. These artifacts may conceal tissue structures and may lead to incorrect medical diagnosis [1]. The motivating application for the present work is transrectal ultrasound (TRUS) guided prostate cancer brachytherapy, a procedure that entails the permanent implantation of small radioactive capsules through hollow needles into the patient's prostate in order to eradicate the cancer with radiation. During the brachytherapy procedure, the physician uses ultrasound slices to visualize the current position of the needle tip that appears as a bright spot in the image. Section-thickness is a unique problem for TRUS-guided brachytherapy because the needles are perpendicular to the ultrasound image slice. The nonuniform section-thickness causes error in localizing the needle tip and thus lead to inaccurate needle placement and ultimately to suboptimal deposition of the radioactive dose. The

G. Fichtinger, A. Martel, and T. Peters (Eds.): MICCAI 2011, Part I, LNCS 6891, pp. 299-306 2011. (C) Springer-Verlag Berlin Heidelberg 2011 


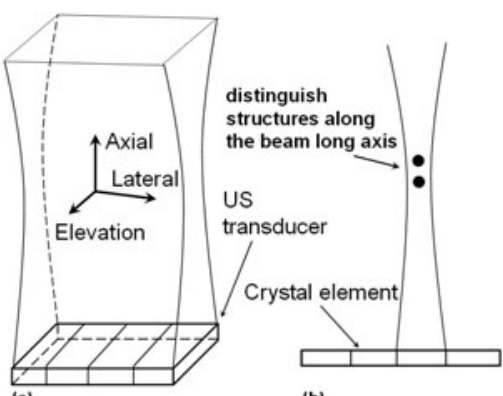

(b)

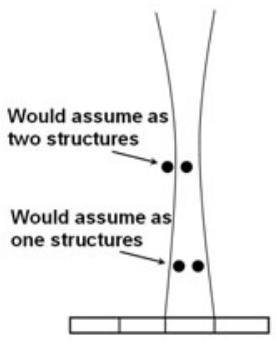

(c)

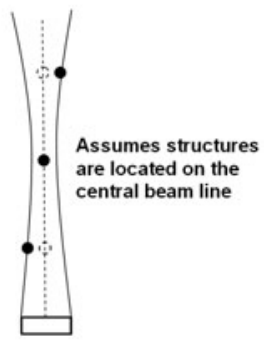

(d)

Fig. 1. (a) US beam pattern in Axial, Lateral, and Elevation axes. (b) Axial resolution, (c) Lateral resolution, and (d) Elevation resolution

objective of this paper is to quantify the needle tip placement error in during the brachytherapy procedure.

The quality of an US image primarily depends on three factors. Axial and lateral resolutions, which is the ability of the US device to distinguish between two structures along the axial and lateral direction, respectively. The third factor is the so called elevation resolution, where the US device assumes that all received echoes originate from structures situated precisely on the central line of the US beam [6], as depicted in Fig. 11 and in more details in Fig. 2. Figure 2(a) and (b) show the cross-section of an unfocused beam pattern and its corresponding Amode echoes generated by reflecting materials in the medium. The first reflected beams, A-C, correspond to the three objects located at the same depth (D) from the transducer within the near-field of the US beam. Since the strength of the US beam is at its peak at the center and it decreases gradually toward the side edges of the beam [1], the maximum echo amplitude (A) corresponds to point $\mathrm{A}$ on the central beam line and the minimum echo corresponds to point $\mathrm{C}$ near the side edges of the beam. The same concept applies to reflecting objects DF, except that the overall US beam intensity decreases farther away from the transducer. Since echoes from the same axial distance and lateral position of the US beam are received by the transducer at the same time, they are absorbed simultaneously. All echoes received at the same time are summed and interpreted as a single object located on the US central beam line [1]. As a consequence, echoes from an object located along the side edge of the US beam appear to be originating from a non-existing virtual reflector on the central region of the US beam, shown as point G in Fig. 2(b). Putting it simply, reflectors along the side edge of the beam do not appear at their true position.

The US section-thickness has been measured using phantoms incorporating inclined surfaces and multiple filaments [1-4]. Recently, we have constructed a device to measure the section-thickness of side firing TRUS probes [5]. We used the same replica of this device for measuring needle localization and placement 

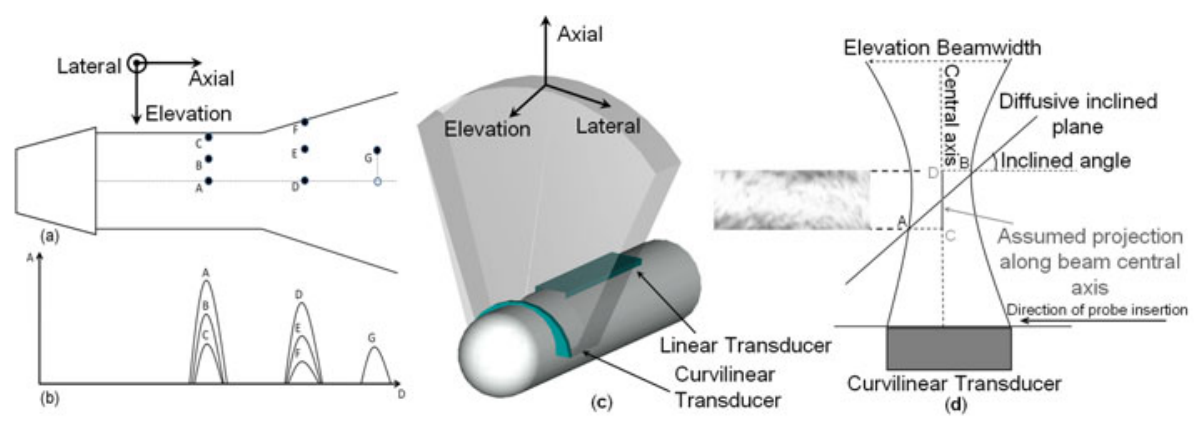

Fig. 2. (a)-(b) Beam pattern of an unfocused B-mode and A-mode US linear array with corresponding point reflectors [1]. (c) Axial, lateral and elevational axes convention with respect to the TRUS beam pattern. (d) Section-thickness estimation principle.

errors caused by the section-thickness of the TRUS beam in a standard clinical brachytherapy setup. To the best of our knowledge there has been no published work on this subject in the open literature.

\section{Methodology}

\subsection{Experimental Setup}

To generate a beam profile for the TRUS probe at $6 \mathrm{MHz}$ central frequency, we moved the probe back and forth (along the elevation axis) to acquire US images of the inclined-plane device (Fig. 3(a)). This is performed when the TRUS probe transducer and profiling phantom were inserted into a clear water bath.

To observe needle tip effects (appeared as bright spots in Fig. 4(c)) on the US images, 6 needles were inserted through holes of a brachytherapy stepper grid template as shown in Fig. 3(b). The needles were placed at the grid's central holes to ensure the distorted parts of the image (along the sides) does not have any influence in our measurements. For each needle, we moved the TRUS probe back and forth (along the elevation axis) in a water bath until the reflection from the needle tips appear on the US image as they intersect with the US beam's boundary. The corresponding probe depth were then recorded for further analysis.

\subsection{US Beam Profile Estimation}

To have an estimate of localization error for a TRUS probe, the US main beam thickness for all depths of the US beam (effective imaging region) from the transducer is found. We do this for the curvilinear transducer by using the same principle first explained in [1]. According to this approach (Fig. 2(d)), as the US beam propagates through the medium it first intersects with point A (nearest point to the transducer) on the inclined diffusive surface ( $45^{\circ}$ to the beam). Similarly, the last point with which the US beam intersects would be the point 


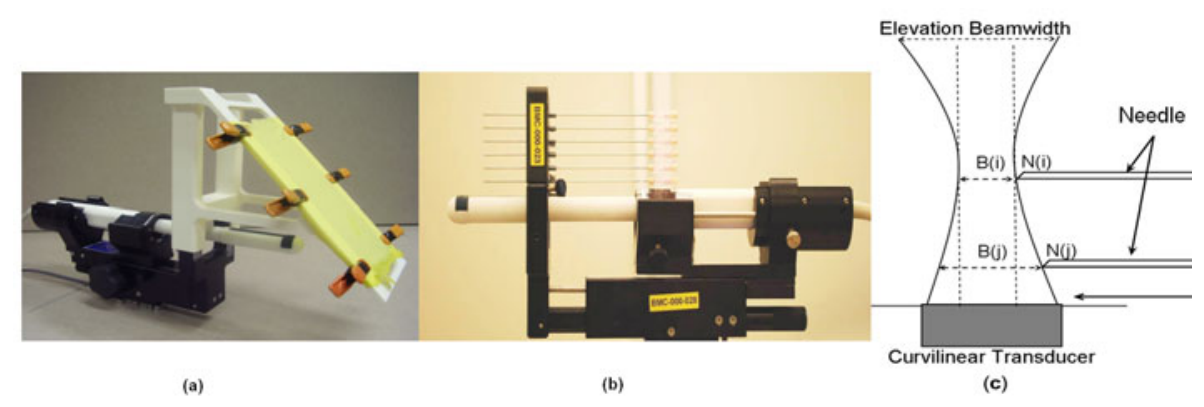

Fig. 3. (a) The beam profiling phantom mounted on a Burdette Medical Systems stepper, (b) Needle insertion setup, (c) Needle offset measurement principle

B (farthest point to the transducer). The sound echoes return to the transducer, however, since the US device assumes all the echoes received to the transducer along the elevation axis of the beam are from the structures on the beam's central line, the line AB would be assumed as line CD to the device. Hence the TRUS image would include a thick bright band which the thickness approximately represents the US main beam thickness. A set of TRUS images with different imaging parameters were collected at $6 \mathrm{MHz}$ for all depths of the imaging region. The artifacts (bright bands) were segmented manually from the images and their distances to the US transducer (position of the band) were taken as the depth measurements. A beam profile of US main beam thickness versus axial distance from the transducer is then plotted as shown in Fig. 4(b) and Fig. [5(a),(c), and (e). Figure 4(a) shows a subset of these measurements.

\section{$2.3 \quad$ Needle Offset Measurement on US Images}

To calculate the needle insertion offsets for every experiment, we set the closest needle to the transducer (needle No. 1) as the reference needle and subtract the depths of the other observed needle positions from that of the reference needle, as shown in Fig. 3(c). The subtracted values represent the amount of divergence or convergence of the beam pattern with respect to the reference needle. Hence the section-thickness relationship between every two inserted needles is defined as:

$$
B(j)=|(N(j)-N(i))| \times 2+B(i)
$$

Where $i$ and $j$ are the two axial depths where the needles are inserted; $N(i)$ and $N(j)$ are the needle insertion depths at axial depths $i$ and $j$ respectively; and $B(i)$ and $B(j)$ are the US main beam thickness at the corresponding $i$ and $j$ axial depths respectively.

\section{Results and Discussion}

In order to observe the effects of the US device imaging parameters on the beam pattern we performed a series of needle insertion tests for 27 combinations of US 


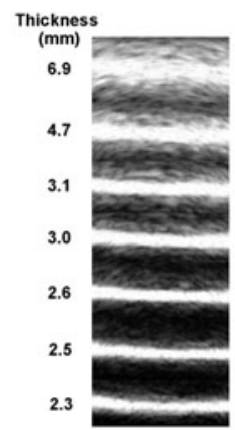

(a)

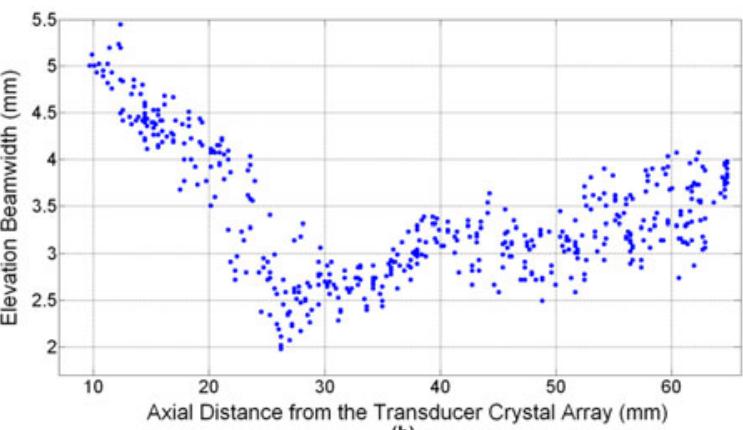

(b)

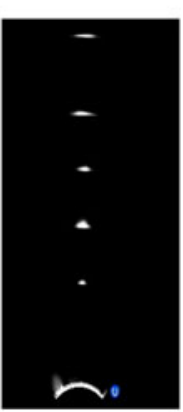

(c)

Fig. 4. (a) A subset of elevation main beam thickness measurements from $28 \mathrm{~mm}$ to $90 \mathrm{~mm}$ axial distance, at gain $=55 \%$, dynamic range $=78 \mathrm{~dB}$, and power $=0$. (b) TRUS elevation beam profile at $6 \mathrm{MHz}$ central operating frequency from $10 \mathrm{~mm}$ to $65 \mathrm{~mm}$ axial distance, at gain $=55 \%$, dynamic range $=78 \mathrm{~dB}$, and power $=0 .(\mathrm{c})$ Needle tip appearance in a TRUS image at gain $=0 \%$, dynamic range $=15 \mathrm{~dB}$, and power $=-7$.
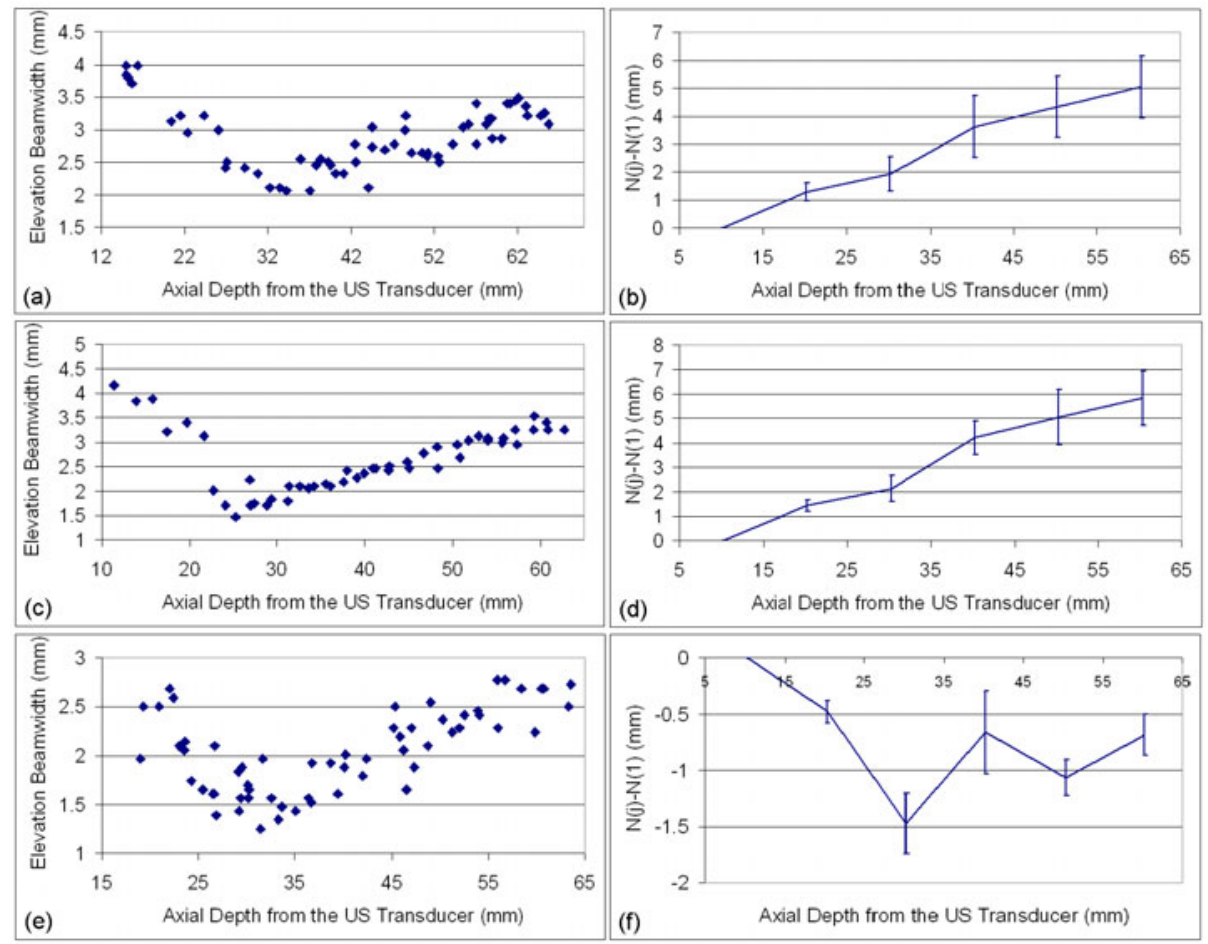

Fig. 5. (a) US beam profile for gain $=100 \%$, dynamic range $=50 \mathrm{~dB}$, and power $=0$. (b) Average and std. of $N(j)-N(1)$ for gain $=100 \%$. (c) US beam profile for gain= $50 \%$, dynamic range $=50 \mathrm{~dB}$, and power $=0$. (d) Average and std. of $N(j)-N(1)$ for gain $=50 \%$. (e) US beam profile for gain $=0 \%$, dynamic range $=50 \mathrm{~dB}$, and power $=0$.

(f) Average and std. of $N(j)-N(1)$ for gain $=0 \%$. 
Table 1. Summary of the needle insertion offsets for different US settings

\begin{tabular}{|c|c|c|c|c|c|c|c|c|}
\hline \multirow{2}{*}{ Gain (\%) } & \multirow{2}{*}{ Dyn. Range (dB) } & \multirow{2}{*}{ Power } & \multicolumn{6}{|c|}{$\mathrm{N}(\mathrm{j})-\mathrm{N}(\mathbf{1})(\mathrm{mm})$} \\
\hline & & & $\mathrm{j}=1$ & $\mathrm{j}=2$ & $j=3$ & $j=4$ & $j=5$ & $j=6$ \\
\hline \multirow{9}{*}{$\mathbf{0}$} & \multirow{3}{*}{15} & $\mathbf{0}$ & 0 & -0.354 & -1.134 & 0 & -0.992 & -0.567 \\
\hline & & -4 & 0 & -0.354 & -1.204 & -0.07 & -1.134 & -0.637 \\
\hline & & -7 & 0 & -0.496 & -1.13 & -0.921 & -1.063 & -0.708 \\
\hline & \multirow{3}{*}{50} & 0 & 0 & -0.496 & -1.559 & -0.637 & -1.275 & -0.779 \\
\hline & & -4 & 0 & -0.567 & -1.417 & -0.85 & -1.134 & -0.637 \\
\hline & & -7 & 0 & -0.496 & -1.559 & -0.637 & -1.275 & -0.779 \\
\hline & \multirow{3}{*}{100} & 0 & 0 & -0.425 & -1.488 & -0.992 & -1.134 & -1.063 \\
\hline & & -4 & 0 & -0.567 & -1.701 & -0.851 & -1.134 & -0.567 \\
\hline & & -7 & 0 & -0.637 & \begin{tabular}{|l|}
-1.77 \\
\end{tabular} & -0.708 & -0.708 & -0.425 \\
\hline \multirow{9}{*}{50} & \multirow{3}{*}{15} & $\mathbf{0}$ & 0 & 1.913 & \begin{tabular}{|l|}
3.047 \\
\end{tabular} & 5.174 & 6.449 & 7.796 \\
\hline & & -4 & 0 & 1.488 & \begin{tabular}{|l|l|}
1.913 \\
\end{tabular} & 4.748 & 5.17 & 6.024 \\
\hline & & -7 & 0 & 1.204 & \begin{tabular}{|l|l|}
2.26 \\
\end{tabular} & 4 & 4.181 & 6.024 \\
\hline & \multirow{3}{*}{50} & 0 & 0 & 1.771 & 2.835 & 5.315 & 6.024 & 7.016 \\
\hline & & -4 & 0 & 1.913 & \begin{tabular}{|l|}
2.551 \\
\end{tabular} & 4.536 & 5.528 & 6.591 \\
\hline & & -7 & 0 & 0.992 & \begin{tabular}{|l|}
2.197 \\
\end{tabular} & 1.913 & 2.976 & 4.181 \\
\hline & \multirow{3}{*}{100} & 0 & 0 & 1.488 & \begin{tabular}{|l|}
2.338 \\
\end{tabular} & 4.6 & 5.244 & 6.237 \\
\hline & & -4 & 0 & 1.488 & \begin{tabular}{|l|}
2.409 \\
\end{tabular} & 4.394 & 4.465 & 6.166 \\
\hline & & -7 & 0 & \begin{tabular}{|l|l|}
1.204 \\
\end{tabular} & \begin{tabular}{|l|l|}
1.771 \\
\end{tabular} & \begin{tabular}{|l|l}
3.827 \\
\end{tabular} & 6.449 & 5.741 \\
\hline \multirow{9}{*}{100} & \multirow{3}{*}{15} & 0 & 0 & \begin{tabular}{|l|l}
1.134 \\
\end{tabular} & 2.055 & \begin{tabular}{|l|}
4.394 \\
\end{tabular} & 5.244 & 5.457 \\
\hline & & -4 & 0 & \begin{tabular}{|l}
1.134 \\
\end{tabular} & \begin{tabular}{|l|}
2.126 \\
\end{tabular} & \begin{tabular}{|l|l}
3.402 \\
\end{tabular} & 4.252 & 4.748 \\
\hline & & -7 & 0 & \begin{tabular}{|l}
1.275 \\
\end{tabular} & 1.7 & \begin{tabular}{|l|l}
2.551 \\
\end{tabular} & 2.976 & 4.394 \\
\hline & \multirow{3}{*}{50} & 0 & 0 & \begin{tabular}{|l|l}
1.488 \\
\end{tabular} & 2.409 & 4.6 & 6.095 & \begin{tabular}{|l}
5.67 \\
\end{tabular} \\
\hline & & -4 & 0 & 1.204 & \begin{tabular}{|l}
1.772 \\
\end{tabular} & 3.118 & 3.402 & 5.174 \\
\hline & & -7 & 0 & \begin{tabular}{|l|l}
1.417 \\
\end{tabular} & \begin{tabular}{|l}
1.134 \\
\end{tabular} & \begin{tabular}{|l}
3.331 \\
\end{tabular} & 3.969 & 3.543 \\
\hline & \multirow{3}{*}{100} & 0 & 0 & 1.13 & 1.488 & 3.685 & 4.394 & 5.032 \\
\hline & & -4 & 0 & \begin{tabular}{|l|l|}
1.204 \\
\end{tabular} & \begin{tabular}{|l}
1.559 \\
\end{tabular} & \begin{tabular}{|l}
4.252 \\
\end{tabular} & 4.252 & \begin{tabular}{|l|}
3.969 \\
\end{tabular} \\
\hline & & -7 & 0 & 1.063 & \begin{tabular}{|l}
0.779 \\
\end{tabular} & 2.551 & 3.402 & 4.04 \\
\hline
\end{tabular}

gain $(0,50$, and $100 \%)$, dynamic range $(15,50$, and $100 \mathrm{~dB})$ and power $(0,-4$, and -7$)$. The results are shown in Fig. 5 and Table 1.

According to Fig. [5(b)-(d), when gain $=50 \%$ or gain $=100 \%$, the needle offsets are monotonously increasing, which indicates the beam diverges constantly. However, this is not the case when comparing with the beam profile generated at the same settings (Fig. 5)(a)-(c)). The beam profile pattern shows that the US beam converges up to a focal point and diverges right after that. This contradiction in the beam profile and needle insertion offset plots could be because of the US side lobes artifacts.

Side lobes consist of multiple low-intensity off-axis ultrasound beams that produce image artifacts due to the error in localizing the returning echoes within the main US beam [6, 7]. If a highly reflective structure is encountered, it will be wrongly positioned in the image along the main US beam [6]. When the gain is set to high, the energy assigned to the US side lobes increases and hence their effects on the TRUS images increase. Hence, during needle insertion, the needle tips are first intersect with the side lobe energies and their echo artifacts are shown as if they are intersected with the main US beam. This clearly shows that the US main lobe thickness and the side lobe artifacts together might have large effects on localizing needle tips and objects within the TRUS images.

On the other hand, when gain $=0 \%$, the side lobe energies are set to be minimum and the needle offsets are less than zero (Fig. $5(f)$ ) which indicates that the 
beam constantly converges up to a focal zone (around $30 \mathrm{~mm}$ from the transducer). The beam pattern starts diverging quickly after the focal zone which matches the main beam thickness measurements using the profiling phantom shown in Fig. 5(e). This indicates that the US section-thickness is small and hence the section-thickness must not introduce much error in the images captured in this setting. On the other hand, when gain is set to zero, the amount of false reflections due to the US side lobes are minimized and hence the needle tip reflections on the TRUS images are ensured to be due to intersecting the needle tips and US main beam only.

\section{Conclusion}

To the best of our knowledge there has been no previous work to examine the effects of imaging parameters and the US section-thickness on needle insertion depth estimation error. The US section-thickness is the combination of the both main sound-energy lobe (the main beam) and the side energy lobe. The side lobe artifacts maybe an important issue to be addressed during needle insertion procedures since they may introduce further localization errors beyond the main beam thickness artifacts.

Both the beam profile and the needle insertion experiments have provided evidence that the high-gain in US imaging setting would increase the side lobe energy of the US beam. This could result in a large elevation section-thickness profile, which directly leads to larger errors in needle insertion for TRUS-guided brachytherapy.

It is highly recommended to reduce the gain of the US imaging device to as low as practically possible to suppress the side lobe-introduced section-thickness, which would effectively minimize the needle insertion depth estimation errors (up to around $2.5 \mathrm{~mm}$ ). The US beam profile also could help the surgeon in considering the likelihood of position errors during needle insertions.

The proposed technology is indeed tailored to brachytherapy, but the underlying principle applies to three-dimensional localization in US imagery, in general, as the US section-thickness is inherent to the modality. Side-lobes artifacts are present in every application where a needle (catheter, etc.) penetrates the US beam in the elevational direction which occurs quite ubiquitously.

Acknowledgements. This work was funded by the Natural Sciences and Engineering Research Council of Canada under the Idea to Innovation program. Gabor Fichtinger was supported as Cancer Care Ontario Research Chair. Thomas $\mathrm{K}$ Chen was supported as a MITACS Accelerate PhD Fellow.

\section{References}

1. Goldstein, A., Madrazo, B.L.: Slice Thickness Artifacts in Gray-Scale Ultrasound. Journal of Clinical Ultrasound 9, 365-375 (1981)

2. Skolnick, M.L.: Estimation of Beam Width in the Elevation (Section Thickness) Plane. Radiology 108, 286-288 (1991) 
3. Richard, B.: Test Object for Measurement of Section Thickness at Ultrasound. Radiology 221, 279-282 (1999)

4. Chen, T.K., Thurston, A.D., Moghari, M.H., Ellis, R.E., Abolmaesumi, P.: A RealTime Ultrasound Calibration System with Automatic Accuracy Control and Incorporation of Ultrasound Section Thickness. In: SPIE Medical Imaging (2008)

5. Peikari, M., Chen, T.K., Burdette, C., Fichtinger, G.: Section-Thickness Profiling for Brachytherapy Ultrasound Guidance. In: SPIE Medical Imaging (2011)

6. Hedrick, F.W., Hykes, D.L.: Ultrasound Physics and Instrumentation. Elsevier Mosby, Missouri (2004)

7. Laing, F.C., Kurtz, A.B.: The Importance of Ultrasonic Side-Lobe Artifacts. Radiology 145, 763-768 (1982) 